\title{
Rhythmic ability decline in aging individuals: The role of movement task complexity
}

\author{
Flora lannarilli, Giuseppe Vannozzi, Carlo Minganti, Laura Capranica, Caterina Pesce \\ Department of Movement, Human and Health Sciences, University of Rome "Foro Italico", Italy
}

\begin{abstract}
Study aim: To investigate age-related changes in rhythmic reproduction ability in relation to the complexity of the adopted movement task.

Material and methods: A Stereophotogrammetric system was used to quantify individual rhythmic performances through motion analysis. Seventeen younger adult (age: $34.8 \pm 4.2 \mathrm{yrs}$ ) and sixteen older adult (age: $69.9 \pm 3.8$ yrs) sedentary individuals volunteered for this study. Participants were administered a rhythmic test, which included three different rhythmic patterns to be reproduced by means of finger-tapping, foot-tapping and walking. Number of correct reproductions, time delays and rhythmic ratios were assessed and submitted to analysis of variance.

Results: For all rhythmic parameters, age-related differences emerged about rhythmic patterns and motor tasks. Older adults showed reduced accuracy as compared to their younger counterparts with a marked tendency to speed up beats reproduction $(\mathrm{p}<0.05)$. Increased movement complexity negatively influenced rhythmic ability, with worst performances in the walking task $(\mathrm{p}<0.05)$.

Conclusions: Complexity of the motor reproduction worsen rhythmic ability. Future research should focus on how specific rhythmic training with progressive movement task complexity could contrast this age-related decline.
\end{abstract}

\section{Keywords: Gait analysis - Tapping - Rhythmic reproduction - Coordination - Elderly}

\section{Introduction}

The performance of many everyday activities typically includes defined sequences of movements and underlying muscular activities according to spatial and temporal constraints $[13,47]$. As age increases from adulthood to elderly, this ability to coordinate spatial and temporal movement parameters deteriorates, with a consequent weakening of the individual's performance in activities of daily living.

Motor performance deficits in older adults include coordination difficulty, slowness of movements and difficulties with balance and gait [49]. Such changes are often linked to a progressive tendency to become sedentary and avoid physical activity, thus leading to a vicious circle that translates into decreased quality of life [35].

Rhythmic ability can play a fundamental role in coordinating movements in a proper order and timing [64]. However, it is not a unitary construct, as it can be conceptualized as a multidimensional skill set reflected in task performances ranging from simply drumming to an isochronous beat to remembering and reproducing complex rhythmic patterns [57]. Overall, it can be defined as the capability of an individual to attend to rhythmic patterns and translate them in a corresponding motor behaviour [15]. In particular, rhythmic ability involves perceptual-cognitive abilities to identify, interpret, and establish the actual relationship among events separated in time [33, 60], and motor coordination abilities to produce responses with appropriate spatiotemporal parameters [40].

Rhythmic ability varies across healthy individuals [19] and its development/decline during the lifespan reflects the combination of maturation, experience and aging processes $[9,20,23,2943,61]$. In particular, the decline due to aging and/or geriatric diseases is related to the well-recognized phenomenon of "generalized slowing" [4, 29, 52]. This has been consistently observed in a range of different motor tasks, such as bimanual tapping [62] and walking [37]. The phenomenon of age-related slowing includes postulates regarding the potential slowing of an internal clock responsible for age-related changes in timing processes of 
rhythmic attending [10]. In complex rhythm production, however, not only such low-level timings are needed. Also, high-level cognition, the executive, which undergoes agerelated decline, is required to exert control over mental sets that specify the overall duration of an action, the number and sequence of its behavioural components [31]. Relatedly, several motor performance aspects [such as coordination difficulties, loss of muscle strength, reduced joint mobility] represent further forms of impairment, playing a role in rhythm reproduction ability. These age-related changes pose issues of accuracy in rhythmic pattern reproduction.

As regards the perceptual-cognitive component of rhythm identification, individuals older than 50 years might encounter problems in the recognition of sounds, which cannot always be explained by hearing loss or pathological impairment in recognition of auditory events [39]. Concerning the temporal aspects of the rhythmic response, older individuals produce significantly larger synchronization errors than younger ones, which can be reduced using rhythmic cueing organized in rhythmic patterns to create a viable gestalt [56].

The individual's capability to correctly discriminate and reproduce a sequence of separate sounds within a rhythmic pattern requires the precise timing of intervals, such that their absolute and relative durations as well as their number and sequencing are respected (i.e., number of events and the temporal ratio among them) $[9,23,24,47]$. To discriminate the rhythmic ability of different populations, a large range of pitch intervals and several complex rhythms have been used $[40,58]$. In particular, interval ratios between the events affect the capability of individuals to reproduce rhythmic patterns, with small integer ratios (i.e., 1:2, 1:3, or 1:4) being typically better reproducible with respect to larger (i.e., 1:5) or non-integer $(1: 2.5$, or $1: 3.5)$ ones $[9,11,12,46]$. In fact, during learning or rehearsal, individuals tend to distort large or non-integer ratios, shifting them toward simpler ratios [9, 12, 46, 54]. Furthermore, binary subdivisions are easier to be discriminated and reproduced with respect to ternary subdivisions $[26,41,58]$. Therefore, the interval ratios between events and their degree of redundancy within a rhythm are likely to concur to rhythm reproduction complexity.

The aforementioned rhythmic complexity is actually intertwined with motor complexity in everyday life, since the different rhythmic structures, either spontaneous or superimposed, have to be reproduced using different motor tasks that could involve different body segments (i.e. hands, feet, and whole body). The justification of operationalising movement task complexity by means of different effectors (fingers/feet) and a different involvement of interlimb coordination and balance control (tapping vs walking) relies on the theoretical framework introduced by Wood [63] that considered different components determining task complexity. Specifically, the frameworks "task as behavior requirements" and "task qua task" could be of interested [63]. The first one involves the definition of the requirements for the action to be performed (i.e. foot tapping while following a given rhythm), while the second one determines the different simple or complex acts required to complete the task. In this respect, different characteristics could be identified to define the different pattern complexity, for instance considering the body segments involved in the motor action.

Consistently, rhythmic performance literature has started to consider task complexity through the adoption of different types of movement task in the rhythm reproduction [24]. Furthermore, Ivanenko et al. [25] hypothesized the presence of a modular control of the lower limbs based on different feedback/feed-forward parameters, aimed at giving wide opportunities to generate motor programs related to rhythmic movements. The existence of a general timing process underlying the movements of limbs and body parts has been already hypothesized thirty years ago [27, 28]. Moreover, some authors have proposed that specific timing processes are required when tasks are not dynamically equivalent, such as tapping, clapping, and galloping $[17,45]$.

Therefore, it emerged that different motor tasks have been employed to study how the central nervous system manages the control of more or less complex tasks in relation to temporal constraints $[8,51,65]$. Tapping has been used to evaluate how the central nervous system manages the perception and reproduction of rhythmic patterns [44]. Since tapping mainly relies on wrist-finger motion only, it has been often used in studies comparing the effects of different movement tasks on the individual capability to reproduce rhythmic patterns $[17,24,36,42]$. To manipulate the transduction delay between tapping and its central representation, stepping has been investigated considering the increased neural distance between the effectors and the brain $[1,44]$. Finally, walking has been used to investigate the coordination of body movements under rhythmic constraints $[2,21,24,37,53]$. The adoption of this complex task in rhythmic assessment studies has also a fair relevance in aging people, as it involves dynamic balance demands and fall risk [18].

In summary, it is known that perceptual integration of auditory stimulations is largely preserved in the elderly despite the evident degradation of attentional and sensorimotor neural processes [5]. Nevertheless, aging individuals show compromised rhythm reproduction abilities that influence the performance of common motor tasks involving complex rhythmic patterns [6] and may significantly impact functional ability in daily life activities [14]. While rhythmic ability studies are gaining momentum in the scientific community, the interest is moving toward a more nuanced understanding of task constraints that may challenge the ability of older individuals to cope with rhythmic 
reproduction in activities of daily living. In particular, there is still the need to investigate whether age and relevant task constraints as motor task complexity might affect rhythmic reproduction ability independently or interactively.

Thus, the present explorative study aimed to investigate age-related differences in rhythmic reproduction ability in relation to different rhythmic structures and motor task complexity.

Our hypothesis is twofold. Firstly, older adults are expected to show a reduced rhythmic ability as compared to younger adults, reflected in a loss of accuracy. Different factors of performance accuracy were considered, moving from general correctness of event number reproduction to more nuanced aspects of absolute and relative timing. Secondly, the age-related reduction of rhythmic ability is expected to be larger when the complexity of rhythm and/ or of motor task increases [24].

We hypothesized that the expected moderation of age effects by task complexity could especially emerge in absolute timing due to the age-related generalized slowing $[4,52]$. The results could further our understanding of the deterioration, with advancing age, of the individual capability to perform complex movement tasks under temporal constraints.

\section{Material and methods}

\section{Experimental approach to the problem}

A cross-sectional study was designed to assess age differences in rhythmic ability depending on rhythmic pattern and movement task complexity. It was hypothesized that comparing rhythmic pattern reproductions by finger tapping (i.e., tapping), foot tapping (i.e., stepping) and walking would provide a nuanced evaluation of aging effects on the individual rhythmic ability.

A computer software (Cubase SX3, Steinberg, Hamburg, Germany) was used to synthesize audio stimuli of a rhythmic test [60], which was used to evaluate the rhythmic ability of the participants. In particular, to manipulate the difficulty in rhythmic reproduction, a rhythmic test (itemized in Figure 1) was proposed according to Persichini \& Capranica [40]. The test included three rhythmic patterns (i.e., r1, r2, r3) with different multilevel time hierarchies (i.e., simple time $4 / 4$ to $60 \mathrm{bpm}$, and compound $12 / 8$ to $80 \mathrm{bpm}$ ), different temporal-ratios between intervals (i.e., 1:1, 2:1, 3:1), numbers of events (range: 6-9) and total duration (range: $2252 \mathrm{~ms}-$ $3375 \mathrm{~ms}$ ) [23]. A commercial media player was used to reproduce each audio stimulus during the experimental session. The different degree of complexity of the three rhythmic patterns has been proven appropriate also for aging research [23].

In order to address the question whether older and younger adults differ in rhythmic ability, different parameters of rhythmic performance have to be considered. Beyond the general correctness of event number reproduction, also absolute and relative timing (i.e. speed of execution and temporal ratio among events respectively) may provide a more nuanced picture of age-related changes. Thus, three dependent variables were considered for the evaluation of the individual's ability to reproduce rhythmic patterns $[23,24,40]: 1) \triangle N E$, defined as the difference between the number of events performed by the participant and the number of events in the standard rhythmic pattern; 2) $\Delta \mathrm{T}$, defined as the difference between the duration of the participant's performance, Tm, and that of the standard rhythm pattern, Ts; and 3) R, defined as the difference between the ratio among rhythmic events of the participant's performance, $\Delta \mathrm{tm}_{\mathrm{i}} / \mathrm{Tm}$, and that of the standard, $\Delta \mathrm{ts}_{\mathrm{i}} / \mathrm{Ts}$. This latter ratio was calculated by means of the following formula $[23,24,40]$ :

$$
\mathrm{R}=\sum_{\mathrm{i}=1}^{\mathrm{n}} \frac{\left|\left(\frac{\Delta \mathrm{tm}_{\mathrm{i}}}{\mathrm{Tm}}-\frac{\Delta \mathrm{ts}_{\mathrm{i}}}{\mathrm{Ts}}\right)\right|}{\frac{\Delta \mathrm{ts}_{\mathrm{i}}}{\mathrm{Ts}}} \cdot \frac{1}{\mathrm{n}}
$$

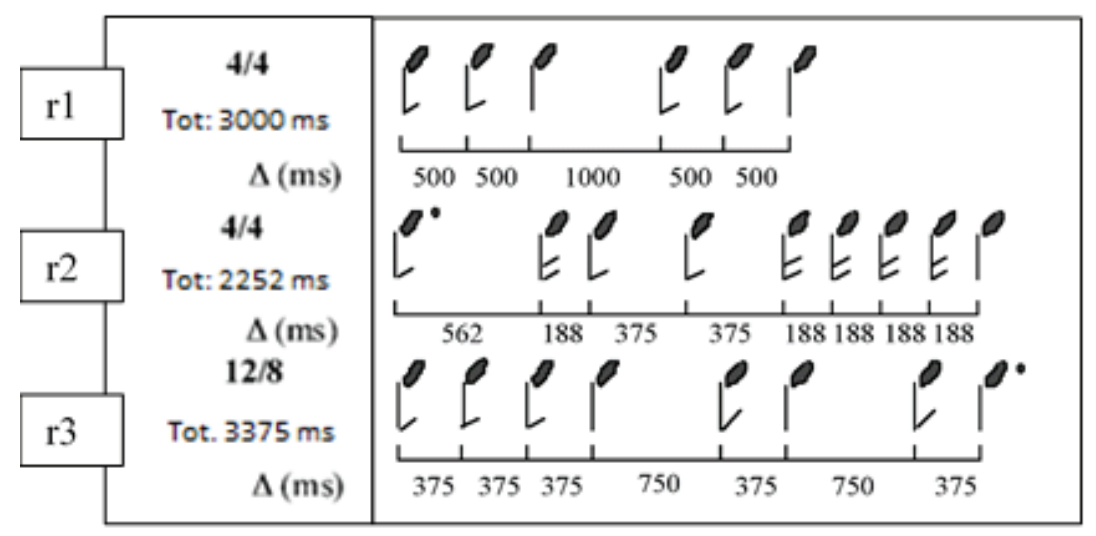

Figure 1. The music notation and inter-event time intervals $(\Delta)$ of the three proposed rhythmic patterns. Adapted from Iannarilli et al. [23] 
The parameters $\Delta \mathrm{T}$ and $\mathrm{R}$ were calculated only if the recorded number of events coincided with that of the standard (correct performance, $\Delta \mathrm{NE}=0$ ). Concerning the temporal parameter, $\Delta \mathrm{T}=0$ represents the perfect reproduction with respect to the standard rhythmic pattern, while $\Delta \mathrm{T}>0$ and $\Delta \mathrm{T}<0$ indicate, respectively, a delayed (i.e slow execution) and an anticipated performance (i.e fast execution) with respect to the standard. Finally, R $>0$ indicates the level of variability of the relative intra-beats timing with respect to the standard.

\section{Participants}

Sixteen older adults ( $8 \mathrm{M}, 8 \mathrm{~F}$; age range: $64-75$ yrs; mean $69.9 \pm 3.8 \mathrm{yrs}$ ) volunteered for this study, after signing a consent form. All the experimental procedures were in accordance with the ethical standards of the local IRB of University of Rome "Foro Italico" and with the 1964 Helsinki declaration and its later amendments or comparable ethical standards. As exclusion criterion, participants should not be rhythmically trained or musicians as this aspect was shown to influence the assessment of rhythmic ability and the relevant data interpretation [23]. Criteria for inclusion in the study were: 1) no formal musical or rhythmic training and no competitive sports activity; 2) absence of neuromuscular and perceptual disorders; and 3) no assumption of medication that could impair cognitive and motor performances. All the participants were healthy individuals with no history of neurological disease, learning disability or impairments of an acoustic or visual nature, as stated by themselves when filled in the section Medical/ Exercise Assessment of AAHPERD Questionnaire [38]. In fact, AAHPERD exercise/medical history questionnaire [38] was administered to ascertain their activity level, educational background, smoking and dietary habits, medication use and history and physical activity. Furthermore, consistently with the guidelines of the ACSM [16], all the involved participants presented a moderate exercise frequency (about $20 \mathrm{~min}$ per day).

For the sake of comparisons, a dataset of adults $(9 \mathrm{M}$, $8 \mathrm{~F}$; age range: $28-44 \mathrm{yrs}$; mean $34.8 \pm 4.2 \mathrm{yrs}$ ) was used [24], complying with the aforementioned inclusion criteria.

\section{Apparatus}

A 9-camera Vicon MX stereophotogrammetric system

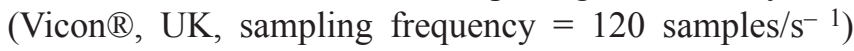
was used to reconstruct the 3D position of 6 retro-reflective spherical skin markers [3], located on the following anatomical landmarks: left and right second finger nail of the hand, left and right metatarsal heads and heels of the foot. The kinematic information was processed aiming at extracting temporal values from which the main parameters were estimated using the software MATLAB (The Mathworks Inc, MA, USA).

\section{Procedures}

According to the literature $[23,24,40]$, participants familiarized with the experimental procedures.

Three motor tasks were employed. (1) Finger-tapping (T): participants, sat in front of a table, performing a tapping task on the table surface by alternating consecutive right and left index fingers. The adoption of the alternated tapping is well represented in the literature [17, 24] as a way to compare with stepping and walking tasks that exhibit the same alternated events. (2) Foot-tapping (i.e. stepping) (S): starting from the upright stance and keeping the same position, the participants reproduced the rhythmic patterns by alternating right and left consecutive foot strikes on the floor. (3) Walking (W): starting from standstill, the participants moved the center of mass in the forward direction by alternating consecutive right and left foot strike events while reproducing the required rhythm.

To facilitate the recognition of new sounds and the control of the subsequent reproductions, participants were allowed to familiarize with the three rhythms as well as with the motor tasks required for the rhythm reproduction. Each rhythm was presented three times [33], each separated by a time interval of the same duration of the rhythm itself (e.g. 3000ms for R1, see Figure 1). According with the literature $[40,55]$, at the end of each sequence, the participant was asked to reproduce the rhythm at the same tempo and in the required movement modality (tapping, stepping or walking) [24] in a no-sound condition. Specifically, three trials were performed, each one separated by a self-selected pause in order to correctly separate the different reproduction during the following postprocessing.

For each experimental condition, three trials were carried out and the best correct performance, i.e. having $\Delta \mathrm{NE}=0$ and the lowest rhythmic ratio $\mathrm{R}$, was considered for further analysis.

To avoid learning effects being systematically associated with experimental conditions, the different nine different experimental conditions obtained by combining rhythmic patterns (r1, r2, r3) and reproduction modalities (T, S, W) were identified (i.e.: r1T, r3S, r2W, r3T, r1W, $\mathrm{r} 2 \mathrm{~S}, \mathrm{r} 3 \mathrm{~W}, \mathrm{r} 2 \mathrm{~T}, \mathrm{r} 1 \mathrm{~S})$ and randomized across participants. The participants were tested individually during an experimental session lasting approximately $20 \mathrm{~min}$.

\section{Data reduction}

Starting from the reconstructed marker 3D position, the following time instants were derived: (1) time instants, at which local minimum of the vertical marker displacement was observed; and (2) time instants, at which local maximum of the vertical acceleration was observed in the range of minimum marker height (Figure 2). From these parameters, temporal occurrence of each participant's 


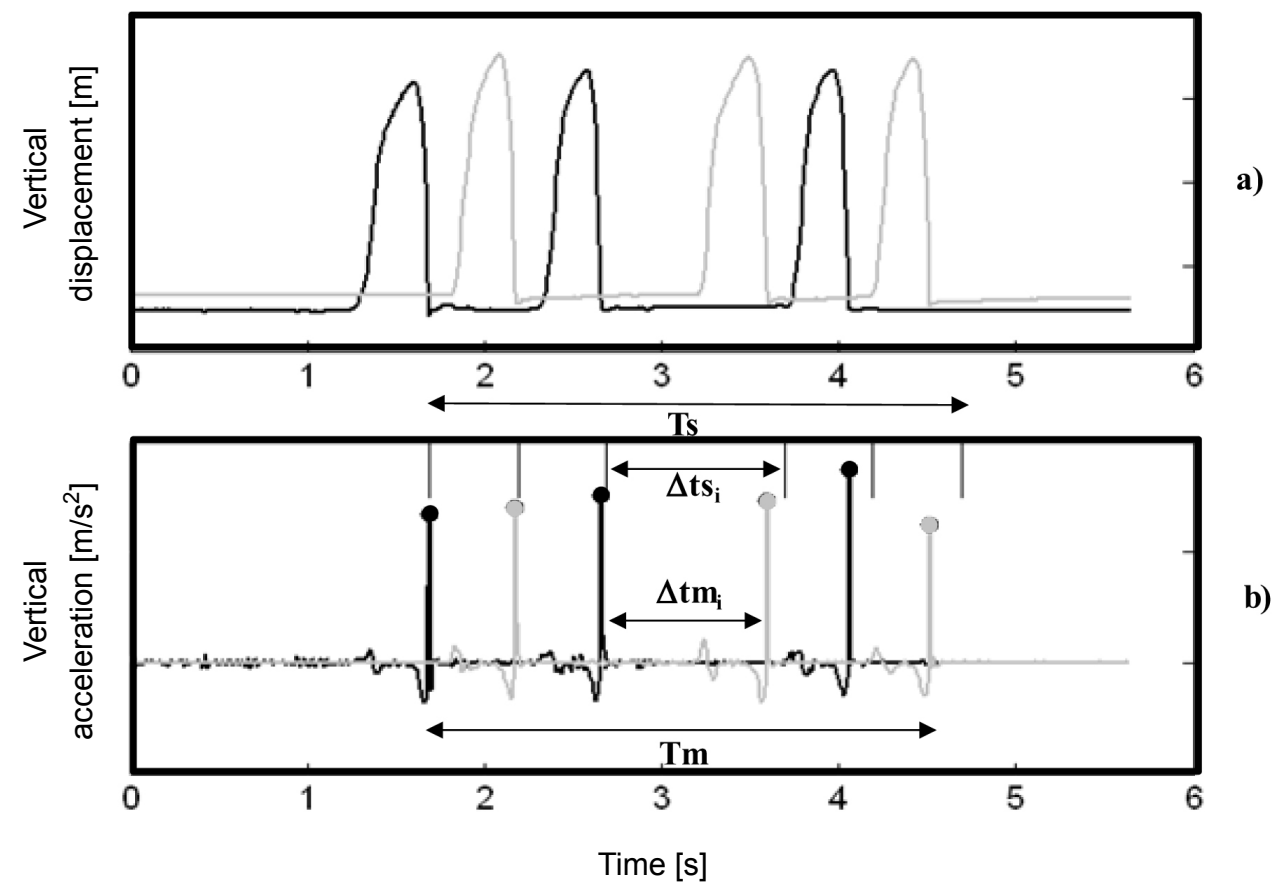

Figure 2. Representation of a tapping reproduction of the first rhythmic pattern: (a) marker vertical position (m); (b) marker vertical acceleration $\left(\mathrm{m} / \mathrm{s}^{2}\right)$. Black line: right finger; grey line: left finger. In the higher part of the b subplot, vertical thin lines and circles indicate the theoretical and the reproduced events, respectively, following the alignment of the first event. Adapted from Iannarilli et al. [24]

event was determined as the time coinciding to the maximum of the vertical acceleration in correspondence of an interval around a minimum marker height (Figure 2). This procedure was designed to prevent possible false positives corresponding to acceleration peaks not matching real beats. For tapping task, each event was estimated considering the marker located on the second fingernail, whereas for stepping and walking tasks, both foot markers were taken into account and the first occurrence of a vertical acceleration peak was considered.

\section{Statistical analysis}

Descriptive statistics, carried out using statistical package SPSS (25.0), were presented as means and standard deviations, and the significance level was set to 0.05 . Normal distribution of the data series was verified using the Shapiro-Wilk test $(\mathrm{p}>0.05)$. A preliminary analysis revealed no gender differences for any of the considered rhythmic performance variables; thus, the gender factor was not further considered.

For each combination of rhythmic patterns $(\mathrm{r} 1, \mathrm{r} 2, \mathrm{r} 3)$ and motor tasks (T, S, W), Chi-Square tests of independence with Yates' correction were performed to examine the associations between correct/wrong performance and age group; when these were found significant, effect sizes were calculated by means of Phi $(\varphi)$ coefficient $(0.1$ small, 0.3 medium, 0.5 large effect).

Only for performances having $\triangle \mathrm{NE}=0$, the parameters $\Delta \mathrm{T}$ and $\mathrm{R}$, calculated for the best performance only, were submitted to analysis. Intraclass correlation coefficients (ICCs) were calculated for $\Delta \mathrm{T}$ and $\mathrm{R}$ values. ICCs lower than 0.60 , ranging from 0.60 to 0.79 or from 0.80 to 1.00 were considered questionable, moderately or highly reliable, respectively. Furthermore, Pearson's correlations between individual's best and average $\Delta \mathrm{T}$ and $\mathrm{R}$ performances were calculated to support best trial selection.

Since only three older adults succeeded in correctly reproducing all three rhythms, $\Delta \mathrm{T}$ and $\mathrm{R}$ parameters were analyzed separately for each rhythmic pattern by means of a $2 \times 3$ mixed ANOVA with repeated measures, considering the age as between factor (younger vs older adults) and the task (i.e. tapping vs stepping vs walking) as within factor. In case of significant interactions, post-hoc comparisons were performed using Fisher's least significant difference test $(p<0.05)$ with Bonferroni correction for multiple comparisons. Furthermore, to provide meaningful analysis for comparisons between small groups, Cohen's effect size (ES) was also calculated for significant effects [7]. An effect size $<0.2$ was considered trivial, from 0.2 to 0.6 small, from 0.7 to 1.2 moderate, and $>1.2$ large. 


\section{Results}

High ICC values for both $\Delta \mathrm{T}$ and $\mathrm{R}$ parameters (higher than 0.91) and Pearson's correlation coefficients (higher than 0.91) supported the selection of the best trial for the further analyses in both groups. As concerns the $\Delta \mathrm{NE}$ parameter, a higher percentage of success of correct reproduction was observed in younger $(76 \%)$ than in older adults $(19 \%)$ for the three tasks and rhythmic patterns. For this reason, the rhythmic patterns were analysed separately (Figure 3$)$. The results showed a difference $(\mathrm{p}<0.05$ with a medium effect, $\varphi$ ranging between 0.4 and 0.6 ) between age groups emerged only for $\mathrm{r} 2$ and $\mathrm{r} 3$. The highest failure frequency between two groups was observed for $\mathrm{r} 2$, with younger adults showing the highest percentage of failure during walking ( $82 \%$ of correct reproductions), whereas older adults during stepping (38\% of correct reproductions).

Regarding the $\Delta \mathrm{T}$ parameter, a main effect for age was found only for $\mathrm{r} 1\left(\mathrm{~F}_{1,94}=19.85, \mathrm{p}<0.001\right.$, ES: 0.40) and r3 $\left(\mathrm{F}_{1,77}=33.92, \mathrm{p}<0.001\right.$, ES: 0.53), with older adults showing anticipated performances with respect to younger adults (see Figure 4).

Total duration also showed a main effect for motor task $\left(\mathrm{r} 1: \mathrm{F}_{2,94}=5.08, \mathrm{p}=0.008\right.$, ES range: 0.03-0.28; $\mathrm{r} 2$ : $\mathrm{F}_{2,68}=18.31, \mathrm{p}<0.001$, ES range: $0.28-0.74 ; \mathrm{r} 3: \mathrm{F}_{2,76}=$ $8.39, \mathrm{p}=0.001$, ES range: 0.05-0.39). Post hoc analysis revealed differences between $\mathrm{W}$ and the other tasks for $\mathrm{r} 1$ $(\mathrm{p}=0.014$ and $\mathrm{p}=0.040$, compared to $\mathrm{T}$ and $\mathrm{S}$ respectively) and for $r 3(p<0.001$ and $p=0.001$, compared to $T$ and $\mathrm{S}$ respectively), with walking always showing a relative delay with respect to the other two tasks. A different result was obtained for $\mathrm{r} 2$, with progressively increasing delays from tapping to stepping and walking and significant differences between each rhythm pair: $T$ vs $S(p=0.006)$, $\mathrm{S}$ vs W $(\mathrm{p}<0.001)$, T vs W $(\mathrm{p}<0.001)$.

For the $\mathrm{R}$ parameter, a main effect for age emerged only for $\mathrm{r} 2\left(\mathrm{~F}_{1,69}=28.73, \mathrm{p}<0.001\right.$, ES: -0.51$)$ and $\mathrm{r} 3$ $\left(\mathrm{F}_{1,68}=8.00, \mathrm{p}=0.006\right.$, ES: -0.30$)$, with older adults showing significantly higher differences than younger adults in the $\mathrm{R}$ values between the standard and the individual reproduction (Figure 5).

A main effect for motor task was observed only for rhythm $\mathrm{r} 1\left(\mathrm{~F}_{2,93}=9.68, \mathrm{p}<0.001\right.$, ES range:0.06-0.41) and $\mathrm{r} 2\left(\mathrm{~F}_{2,68}=5.35, \mathrm{p}=0.007, \mathrm{ES}=0.563\right)$. Post hoc analysis revealed differences between $\mathrm{W}$ and the other tasks both for $\mathrm{r} 1(\mathrm{p}<0.001$ and $\mathrm{p}=0.002$, for $\mathrm{T}$ and $\mathrm{S}$ respectively) and for $\mathrm{r} 2(\mathrm{p}=0.008$ and $\mathrm{p}=0.007$, for $\mathrm{T}$ and $\mathrm{S}$ respectively), with walking showing higher values (17 $\pm 13 \%)$, as compared to stepping $(10 \pm 12 \%)$, and tapping ( $9 \pm 11 \%$; see Figure 5).

\section{Discussion and conclusions}

The present study examined the decline of rhythmic ability in older adults and how this ability is affected by increasing complexity of the rhythm and motor reproduction modality. The main tested hypotheses were: i) older adults present a reduced rhythmic ability compared to younger adults, as reflected in the loss of accuracy in rhythmic performance; ii) this age-related decline is expected to be larger as rhythm and motor task complexities increase.

The results indicated a dramatic reduction in rhythmic ability with advancing age. In fact, while the adults

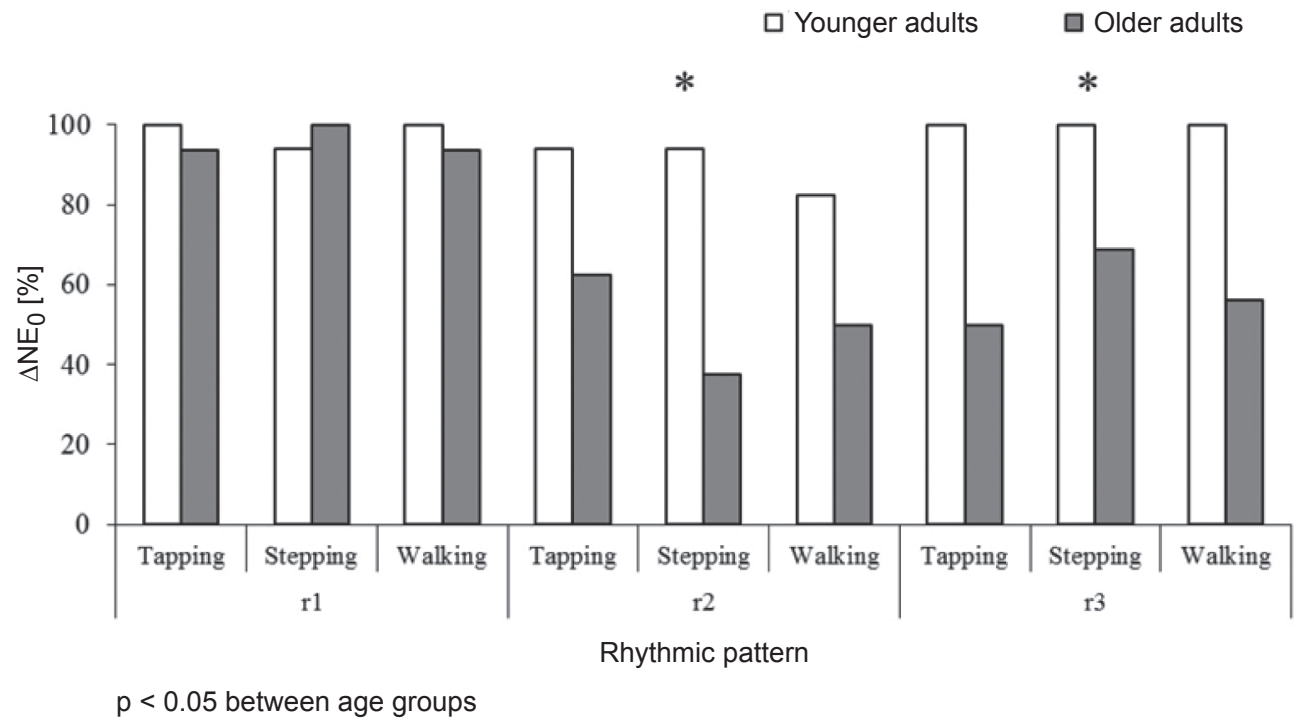

Figure 3. Frequency of occurrence $\Delta \mathrm{NE}_{0}$ of participants correctly reproducing the number of events $(\Delta \mathrm{NE}=0)$ for the three tasks during the three rhythmic patterns. The asterisk refers to a difference between the two age-groups for each motor task $(\mathrm{p}<0.05)$ 

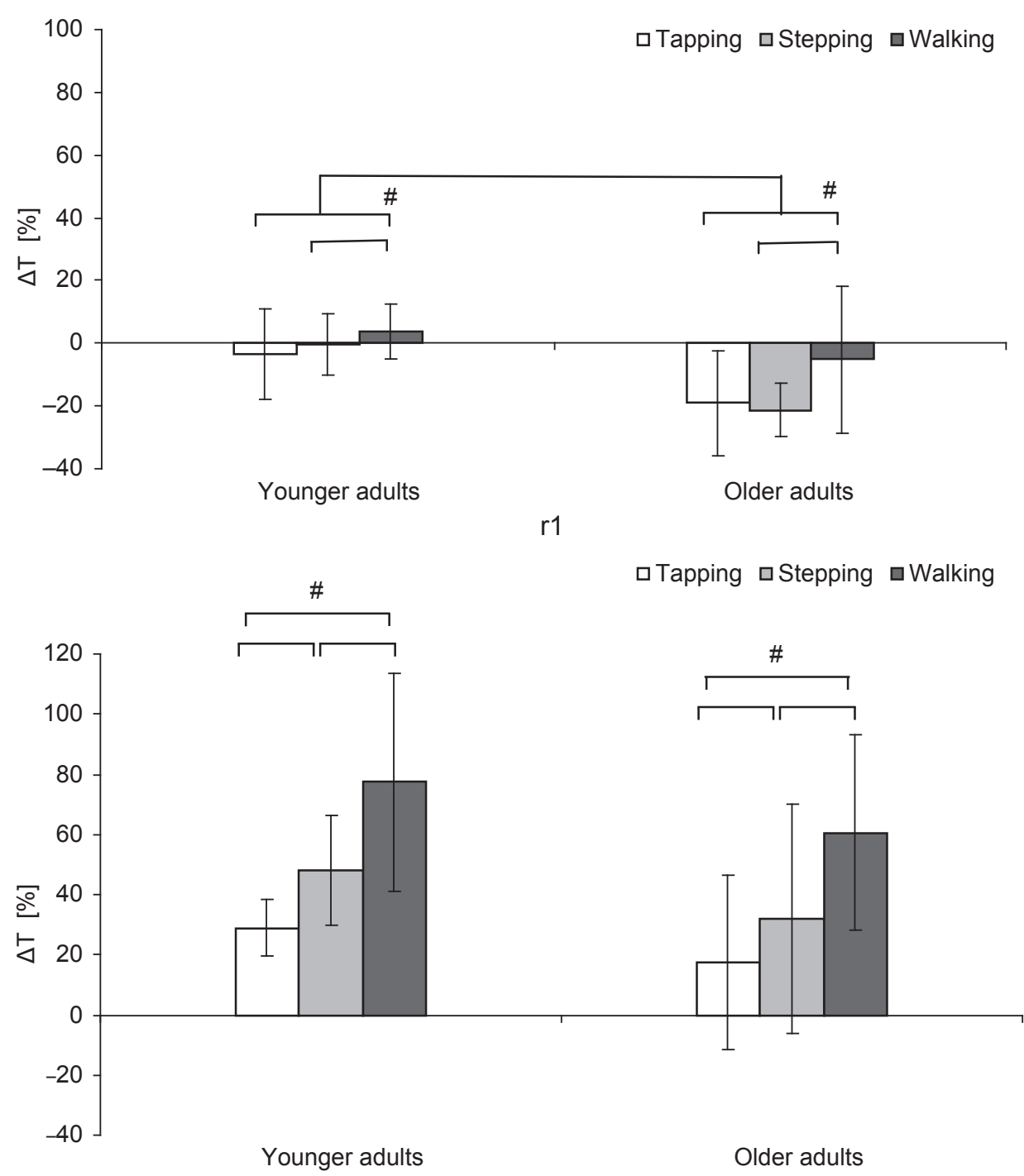

r2

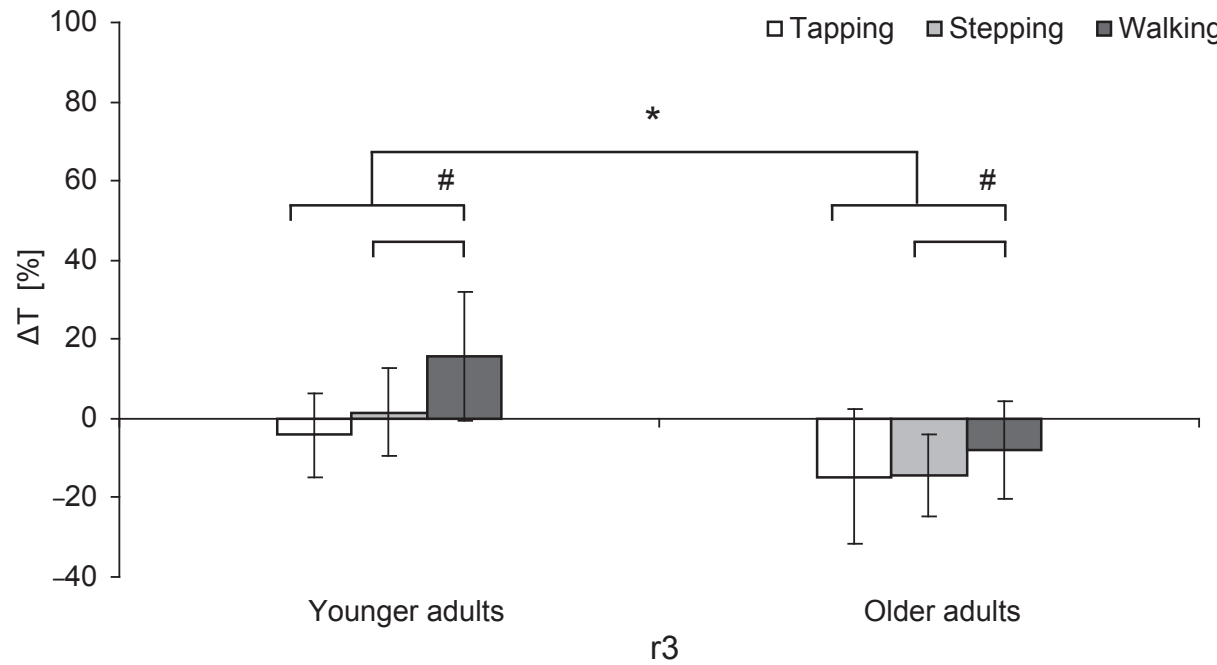

Figure 4. Means and standard deviations of the percentage differences between the duration of subject's performance and that of the standard rhythm pattern for the three tasks during the three rhythmic patterns. Negative values indicate anticipations, positive values delays 

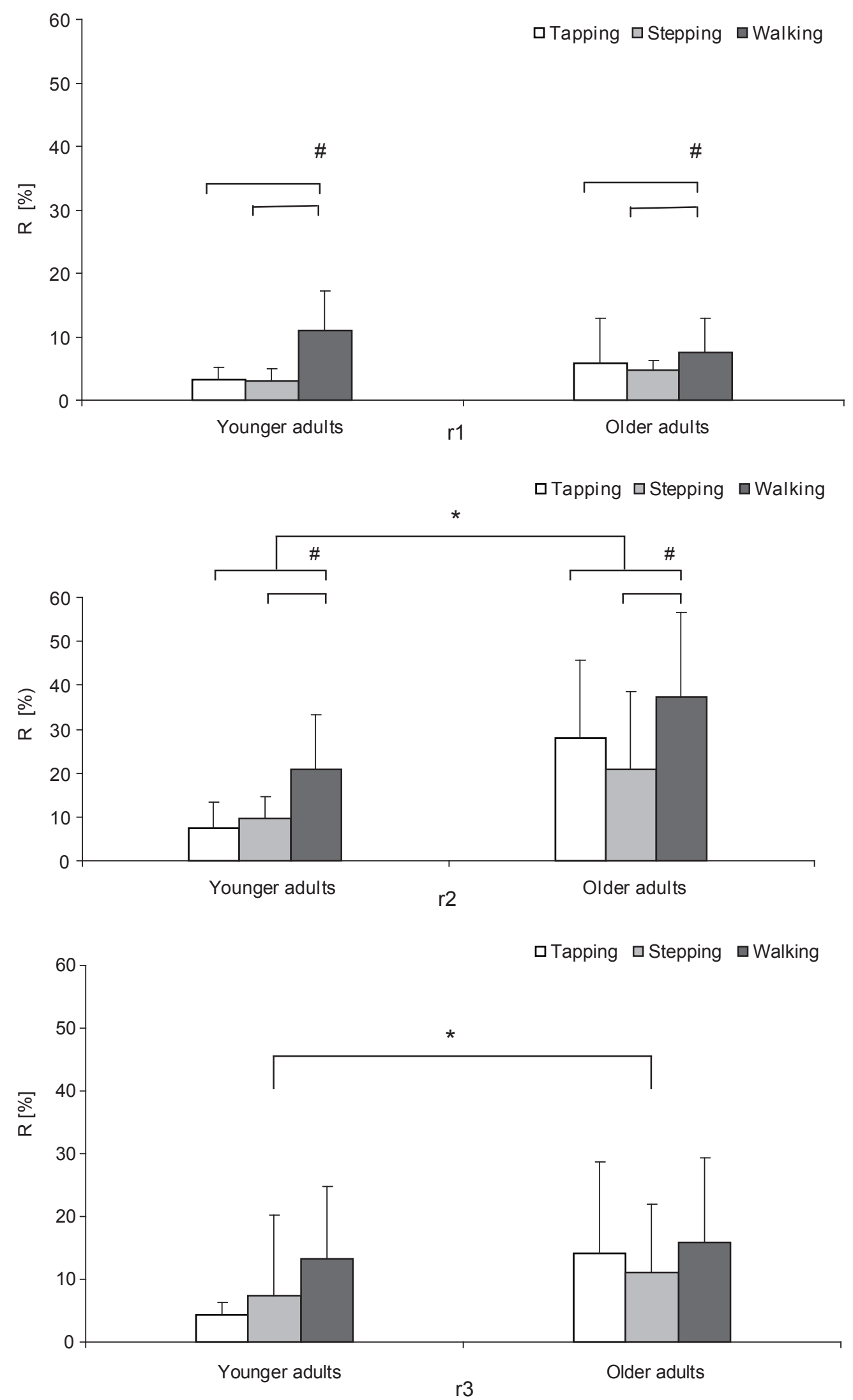

Figure 5. Means and standard deviations of the ratios among rhythmic events $(\mathrm{R})$ for the three rhythmic patterns (r1, r2, and r3) and the three movement tasks (tapping, stepping, and walking) 
showed a good ability to discriminate the number of rhythmic events $(\triangle \mathrm{NE})$, only $19 \%$ of the older participants was able to reproduce all the proper number of events of the three rhythmic patterns with each movement task. Concerning the analysis of each rhythm, age-related deteriorations were observed in the total duration of reproduction patterns and in the rhythmic ratio among beats. In particular, $\Delta \mathrm{T}$ and $\mathrm{R}$ allowed to better detail the role of both rhythm and task complexity in this reduced reproduction performance of both groups. These parameters were actually sensitive to different dimensions of task complexity as referred by Wood [63] with R linked to the complexity of the behavioural responses and rhythmic information cues and $\Delta \mathrm{T}$ by the relationship between them [24].

Specifically, as expected, a progressive performance deterioration was observed in both age groups as rhythm and motor task complexity increased. These findings were in line with the literature $[50,61]$ concerning the agerelated loss of accuracy of physical movements across a variety of daily activities and laboratory tasks. However, contrary to our expectation, the absence of interactive effects between age and movement task complexity for each examined rhythm, suggests that an increase in task complexity does not disproportionally worsen rhythm reproduction performance in older adults compared to younger adults. Speculatively, varying the movement type may not significantly change the demands on executive control, which typically undergoes an age-related decline [32]. Executive control seems to come into play in rhythm production for selecting between competing elements of different rhythms [31] rather than for performing a same rhythm with different effectors and movement types.

Beyond this basic result, the use of different parameters of rhythmic pattern reproduction allowed obtaining a more differentiated and nuanced pattern of age-related changes in rhythmic ability. As for the rhythm complexity, different aspects of temporal or rhythmic structure have been studied by manipulating the time hierarchies (simple 4/4 and compound 12/8 times) and the inter-beat ratios (i.e., 1:1, 2:1, 3:1) of three rhythmic patterns. As expected, $[9,24,58]$, the participants reproduced with higher accuracy the first pattern that includes a regular rhythmic structure (i.e., a reiterated 1:1:2 beat sequence) consistent with its simple time hierarchy (i.e., 4/4). Instead, the longer interval between first two beats in $\mathrm{r} 2$ (i.e., a complex 3:1 ratio) and the following four short interbeat intervals determined that the second pattern $\mathrm{r} 2$ was a non-systematic sequence, inconsistent with the simple 4/4-time hierarchy. Thus, reproducing longer intervals at the beginning of $\mathrm{r} 2$ rhythm production followed by shortduration sixteen notes, determined the highest occurrence of deviation from the standard, making persons in higher difficulty to estimate movement timing. Despite the third pattern presented a compound time (i.e., 12/8), younger adults succeeded in reproducing the number of events, probably due to its component duration related by simple integer-ratios [9].

These findings support the evidence that in younger adults, rhythmic reproduction is more strongly affected by complex and varied temporal intervals than by the time hierarchies of the rhythmic patterns [40,58]. Consistently with what reported in similar domains such as the identification and recognition of sounds [39], difficulty in reproducing the correct number of events for older adults are extended also to simpler rhythmical structures (e.g., the third pattern).

The ability to reproduce the duration of a pattern, absolute timing, and to maintain the time relation among events, relative timing, is crucial for rhythmic proficiency [29]. Although both parameters contribute to the accuracy of rhythmic reproductions, the ability to maintain the relative timing is the most important indicator of rhythmic proficiency, independently of the strict respect of the absolute timing [30].

Concerning the temporal parameter, both age groups showed a progressive reduction of rhythmic performance as rhythm/task complexity increased, as also widely confirmed in the literature about motor control adaptations due to aging [49]. In our study, we observed that older participants were always faster than younger adults in all conditions, independently from rhythm and task complexity (see Figure 4). Specifically, older adults exhibited an anticipated performance $(\Delta \mathrm{T}<0)$ in $\mathrm{r} 1$ and $\mathrm{r} 3$, while they showed a delayed performance $(\Delta \mathrm{T}>0)$ only in $\mathrm{r} 2$. In other words, older adults who succeeded in the reproduction of the correct number of events showed significantly anticipated performances as compared to younger adults, showing the tendency to speed up their actions. These findings shouldn't be read as contrasting with the general age-related tendency to slow down movements [52], and with the slowing-down of the preferred movement frequency as the age increases [59,61], but rather as a way to point out their own strategy to compensate for aging effects. Consistently, Nagasaki et al. (1996) reported, in young-old adults similar to our sample, both faster finger tapping and increased step rate in walking and attributed this phenomenon to a common origin in the brain as observed in Parkinsonian patients.

Considering the effect of aging on total duration and ratio among rhythmic events jointly, older adults seem to prioritize movement speed instead of rhythmic accuracy, whereas the opposite was observed in younger individuals. Furthermore, it is worth considering that two out of three movement tasks (i.e. stepping and walking) involved posture and gait control. In light of this, the present finding about older adults trading rhythmic accuracy for movement speed may reflect a strategy in line with the theory that, when faced with dual cognitive-walking tasks, older 
adults prioritize postural and gait control to avoid threats to balance [48]. Specifically, it is known that the presence of mild cognitive tasks improves postural performance because of a shift of attention away from the highly automatized activity [22]. Therefore, it is not surprising to observe that, when in presence of a less attention-demanding rhythm $(\mathrm{r} 1, \mathrm{r} 3)$, older adults present a better temporal rhythmic performance $(\Delta \mathrm{T})$ while walking. Conversely, when facing a higher level of cognitive demands, a resource competition is set-in. Therefore, when considering the most difficult dual task using $r$, older individuals presented the worst reproduction performance in terms of higher deviation from the standard rhythmic model. Even if this aspect would require further research work with a specific focus of cognitive assessment, we can hypothesize that this decline in performance in the elderly could also depend on reduced capacity of working memory, a system for the temporary storage and manipulation of information, which suffers from age-related degeneration. In fact, elderly people perform disproportionally poor in more complex tasks challenging executive function and especially working memory [34].

The reproduction of rhythmic patterns could also suffer from age-related deteriorations in coordination [52]. In this regard, the comparison between fingers, feet, and whole-body rhythmic performances represents a valuable paradigm to understand the complex relationship between rhythmic recognition and reproduction. Despite a progressive increase in coordination demands was hypothesized to exist from tapping to walking [63] and expected to affect pattern reproduction, this phenomenon clearly emerged only for the total duration of the second rhythm reproduction (see Figure 4) for both age groups. Instead, for the other two rhythms, tapping and stepping showed similar values independently of age while walking presented higher values especially in the elderly. Therefore, concerning the individual's capability to move according to a rhythm, walking resulted to be the most difficult task regardless of age. Probably, the use of different body areas while reproducing rhythms would entail a progressive performance difficulty due to the number of body segments involved, due to the relevant anthropometric quantities (i.e. body mass and lengths), and due to the possible body translation in space that describe a motion trajectory. In fact, age-related physiological changes (such as loss of muscle strength/power, impaired reaction times and reduced joint mobility) could play a role in the outlined worst performance in the walking modality. This could be likely a sign of reduced motor control in the modulation of concentric/ eccentric muscle contractions during the continuous accelerating/braking actions applied to the moving body while walking, so as to comply with the required rhythm constraints. Furthermore, another possible explanation might rely on the fact that individuals spontaneously follow rhythms or music by means of tapping or stepping. Moreover, walking has its own spontaneous rhythm that could prevail over the reproduction of the external rhythm thus leading to a failure in rhythm reproduction. Therefore, as expected, the multi-joint coordination during locomotion determined the largest delay in total duration and the highest difference in rhythmic ratios.

However, a main effect for movement task complexity was found only for the easiest and the most difficult patterns ( $\mathrm{r} 1$ and $\mathrm{r} 2$, respectively), whereas no difference between tasks emerged for the ratio among rhythmic events of the third pattern, characterized by intermediate difficulty level. This aspect could be related to the fact that $r 3$ is a compound ratio that, differently from the other two rhythms, easily leads to continuous movement. However, due to the high variability observed, this initial observation should be corroborated by further research, also taking into account different simple time structures, like isochronous sequences, to support data interpretation and disentangle rhythm and motor complexity. An interesting strength point of this study is represented by the combined use of general, relative and absolute timing data derived from objective measurements of human movement provided by stereophotogrammetry, from which the considered rhythmic parameters were defined.

A number of limitations should be outlined for this study. While several changes were pointed out in the aged group as due to both rhythm and task complexity, a more comprehensive evaluation could be done by verifying further aspects as balance and working memory. These could be possible aspects to quantify to better understand possible sources of the variation in the identified set of results. In this respect, future studies could consider a stratification of older participants through the adoption of cognitive testing and/or using a classification using a balance test.

In conclusion, considering that rhythmic ability underlies performance of several daily tasks, it could be advisable to use these new findings to investigate whether aging individuals could profit from specific training aiming to increase this ability and contrasting the decline due to aging. Based on our results, we may suggest that such training programs could be more effective if tailored on the complexity of both movement task and rhythmic structure, towards an improved quality of life.

\section{Conflict of interest: Authors state no conflict of interest.}

\section{References}

1. Aschersleben G. (2002) Temporal Control of movements in Sensorimotor Synchronization. Brain Cogn., 48: 66-79. DOI: 10.1006/brcg.2001.1304. 
2. Beauchet O., Allali G., Berrut G., Dubost V., Assal F. (2008) Gait analysis in demented subjects: Interests and perspectives. Neuropsych Dis. Treat., 4: 155-160.

3. Benedetti M.G., Beghi E., De Tanti A., Cappozzo A., Basaglia N., et al. (2017) SIAMOC position paper on gait analysis in clinical practice: General requirements, methods and appropriateness. Results of an Italian consensus conference. Gait Posture, 58: 252-260. DOI: 10.1016/j. gaitpost.2017.08.003.

4. Birren J.E., Woods A.M., Williams M.V. (1980) Behavioural slowing with age: Causes, organization, and consequences. In: Poon L.W. (eds.) Aging in the 1980s. DC: American Psychological Association Washington, pp. 293-308. DOI: 10.1037/10050-021.

5. Blais M., Martin E., Albaret J.M., Tallet J. (2014) Preservation of perceptual integration improves temporal stability of bimanual coordination in the elderly: An evidence of age-related brain plasticity. Behav. Brain Res., 275: 34-42. DOI: 10.1016/j.bbr.2014.08.043.

6. Block R.A., Zakay D., Hancock P.A (1998) Human aging and duration judgments: A meta-analytic review. Psychol. Aging, 13: 584-59. DOI: 10.1037/0882-7974.13.4.584.

7. Cohen J. (1988) Statistical power analysis for the behavioural sciences. NJ: Erlbaum, Hillsdale.

8. Delignières D., Lemoine L., Torre K. (2004) Time intervals production in tapping and oscillatory motion. Hum. Mov. Sci., 23: 87-103. DOI: 10.1016/j.humov.2004.07.001.

9. Drake C. (1993) Reproduction of musical rhythms by children, adult musicians, and adult nonmusicians Atten. Percept. Psychophys., 53(1): 25-33.

10. Drake C., Jones M.R., Baruch C. (2000) The development of rhythmic attending in auditory sequence: attunement, referent period, focal attending. Cognition, 77: 251-288. DOI: 10.1016/S0010-0277(00)00106-2.

11. Essens P.J. (1986) Hierarchical organization of temporal patterns. Atten. Percept. Psychophys., 40: 69-73.

12. Essens P.J., Povel D.J. (1985) Metrical and nonmetrical representations of temporal patterns. Percept Psychophys., 37: 1-7.

13. Fairbrother J.T. (2010) Fundamentals of motor behavior. Human Kinetics Champaign.

14. Forte R., Boreham C.A.G., De Vito G., Pesce C. (2015) Health and Quality of Life Perception in Older Adults: The Joint Role of Cognitive Efficiency and Functional Mobility. Int. J. Environ. Res. Public Health, 12: 1328-1134. DOI: 10.3390/ijerph120911328.

15. Fraisse P., Pichot P., Clairouin G. (1949) Les aptitudes rythmiques: Etude comparée des oligophrénes et des enfants normaux. J. Psychol. Norm. Pathol., 42: 309-330.

16. Garber C.E., Blissmer B., Deschenes M.R., Franklin B.A., Lamonte M.J., Lee I.M., Nieman D., Swain D.P. (2011) Quantity and quality of exercise for developing and maintaining cardiorespiratory, musculoskeletal, and neuromotor fitness in apparently healthy adults: guidance for prescribing exercise. Med. Sci. Sport Exerc., 43: 1334-1359. DOI: 10.1249/MSS.0b013e318213fefb.

17. Getchell N., Forrester L., Whitall J. (2001) Individual differences and similarities in the stability, timing consistency, and natural frequency of rhythmic coordinated actions. Res. Q. Exerc. Sport, 72(1): 13-21. DOI: 10.1080/02701367.2001.10608927.

18. Ghai S., Ghai I., Effenberg A.O. (2018) Effect of rhythmic auditory cueing on aging gait: A systematic review and Meta-Analysis. Aging Dis., 9: 901-923. DOI:10.14336/ AD.2017.1031.

19. Grahn J.A., Schuit D. (2012) Individual differences in rhythmic ability: Behavioral and neuroimaging investigations. Psychomusicology, 22: 105-121. DOI: 10.1037/ a0031188.

20. Halpern A.R., Bartlett J.C., Dowling W. (1995) Aging and experience in the recognition of musical transpositions. Psychol. Aging, 10(3): 325-342. DOI: 10.1037/08827974.10.3.325.

21. Hausdorff J.M., Yogev G., Springer S., Simon E.S., Giladi N. (2005) Walking is more like catching than tapping: Gait in the elderly as a complex cognitive task. Exp. Brain Res., 164: 541-548. DOI: 10.1007/s00221-0052280-3.

22. Huxhold O., Li S.C., Schmiedek F., Lindenberger U. (2006) Dual-tasking postural control: Aging and the effects of cognitive demand in conjunction with focus of attention. Brain Res. Bull., 69: 294-305. DOI: 10.1016/j. brainresbull.2006.01.002.

23. Iannarilli F., Pesce C., Persichini C., Capranica L. (2013a) Age-related changes of rhythmic ability in musically trained and untrained individuals. Sport Sci. Health, 9: 43-50. DOI 10.1007/s11332-013-0144-y.

24. Iannarilli F., Vannozzi G., Iosa M., Pesce C., Capranica L. (2013b) Effects of task complexity on rhythmic reproduction performance in adults. Hum. Mov. Sci., 32: 203-213. DOI: 10.1016/j.humov.2012.12.004.

25. Ivanenko Y.P., Poppele R.E., Lacquaniti F. (2006) Motor control programs and walking. Neuroscientist, 12: 339-348. DOI: 10.1177/1073858406287987.

26. Jones M.R. (1987) Dynamic pattern structure in music: Recent theory and research. Percept. Psychophys., 41: 621-634.

27. Keele S.W., Hawkins H.L. (1982) Explorations of individual differences relevant to high level skill. J. Motor Behav., 14: 2-23. DOI: 10.1080/00222895.1982.10735259.

28. Keele S.W., Ivry R.I., Pokorny R.A. (1987) Force control and its relation to timing. J. Motor Behav., 19: 96-114. DOI: 10.1080/00222895.1987.10735402.

29. Krampe R.T. (2002) Aging, expertise and fine motor movement. Neurosci. Biobehav. Rev., 26: 769-776. DOI: 10.1016/S0149-7634[02]00064-7. 
30. Krampe R.T., Engbert R., Kliegl R. (2001) Age-specific problems in rhythmic training. Psychol. Aging, 16: 12-30. DOI: 10.1037/0882-7974.16.1.12.

31. Krampe R.T., Mayr U., Kliegl R. (2005). Timing, Sequencing, and Executive control in repetitive movement production. J. Exp. Psychol. Hum. Percept. Perform., 3: 379-397. DOI: 10.1037/0096-1523.31.3.379.

32. Luszcz M. (2011) Executive function and cognitive aging - Handbook of the psychology of aging, Elsevier. DOI: 10.1016/B978-0-12-380882-0.00004-8.

33. Mc Adams S., Bigand E. (1999) Introduction to auditory cognition. In: McAdams S., Bigand E. (eds.), Thinking in sound: The cognitive psychology of human audition. Clarendon Press, Oxford, pp. 1-10. DOI: 10.1093/acprof: oso/9780198522577.001.0001.

34. Mattay V.S., Fera F., Tessitore A., Hariri A.R., Berman K.F., Das S., Meyer-Lindenberg A., Goldberg T.E., Callicott J.H., Weinberge D.R. (2006) Neurophysiological correlates of age-related changes in working memory capacity. Neurosci. Lett., 392: 32-37. DOI: 10.1016/j. neulet.2005.09.025.

35. Motl R. W., McAuley E. (2010) Physical activity, disability, and quality of life in older adults. Phys. Med. Rehabil. Clin., 21: 299-308. DOI: 10.1016/j.pmr.2009.12.006.

36. Nagasaki H. (1990) Rhythm and variability of timing in periodic tapping. Hum. Mov. Sci., 9: 177-194. DOI: 10.1016/0167-9457(90)90026-A 37.

37 Nagasaki H., Itoh H., Hashizume K., Furuna T. (1996) Walking patterns and finger rhythm of older adults. Percept. Mot. Skills, 82: 435-447. DOI: 10.2466/ pms.1996.82.2.435.

38. Osness W.H., Adrian M., Clark B., Hoeger W., Raab D., Wiswell R. (1996) Functional fitness assessment for adult over 60 years. Kendall/Hunt, Dubuque.

39. Peretz I. (1993) Auditory Agnosia: A functional analysis. In: McAdams S., Bigand E. (eds.) Thinking in sound. The cognitive psychology of human auditions. Oxford Clarendon press, pp. 199-230. DOI: 10.1093/acprof:oso /9780198522577.001.0001.

40. Persichini C., Capranica L. (2004) Reproduction of rhythmic patterns in young individuals: Toward the construction of a computerized rhythmic test. Int. J. Sport Psychol., 35: 246-265.

41. Povel D.J. (1981) Internal representation of simple temporal patterns. J. Exp. Psychol. Hum., 7: 3-18.

42. Rabinowitz I., Lavner Y. (2014) Association between finger tapping, attention, memory, and cognitive diagnosis in elderly patients. Percept. Mot. Skills, 119: 259-278. DOI: $10.2466 / 10.22$.PMS.119c12z3.

43. Reifinger J.L. Jr. (2006) Skill development in rhythm perception and performance: A review of literature. Update: Applications of research in music education. 25(1): 15-27. DOI: 10.1177/87551233060250010103.
44. Repp B.H. (2005) Sensorimotor synchronization: A review of the tapping literature. Psychon. Bull. Rev., 12(6): 969-992.

45. Robertson S.D., Zelaznik H.N., Lantero D.A., Bojczyk K.G., Spencer R.M., Doffin J.G., Schneidt T. (1999) Correlations for timing consistency among tapping and drawing tasks: Evidence against a single timing process for motor control. J. Exp. Psychol. Human., 25: 1316-1330. DOI: 10.1037/0096-1523.25.5.1316.

46. Sakai K., Hikosaka O., Miyauchi S., Takino R., Tamada T., Iwata N.K., Nielsen M. (1999) Neural Representation of a rhythm depends on its interval ratio. J. Neurosci., 19: 10074-10081. DOI: 10.1523/JNEUROSCI.19-2210074.1999.

47. Sakai K., Hikosaka O., Nakamura K. (2004). Emergence of rhythm during motor learning. Trends Cogn. Sci., 8(12): 547-553. DOI: 10.1016/j.tics.2004.10.005.

48. Schaefer S., Schumacher V. (2011) The Interplay between Cognitive and Motor Functioning in Healthy Older Adults: Findings from Dual-Task Studies and Suggestions for Intervention. Gerontology, 57: 239-246. DOI: 10.1159/000322197.

49. Seidler R.D., Bernard J.A., Burutolu T.B., Fling B.W., Gordon M.T., Gwin J.T., Kwak Y., Lipps D.B. (2010) Motor Control and Aging: Links to Age-Related Brain Structural, Functional, and Biochemical Effects. Neurosci Biobehav. Rev., 34: 721-733. DOI: 10.1016/j. neubiorev.2009.10.005.

50. Sleimen-Malkoun R., Temprado J.J., Hong L. (2014) Aging induced loss of complexity and dedifferentiation: consequences for coordination dynamics within and between brain, muscular and behavioral levels. Front. Aging Neurosci., 6: 1-17. DOI: 10.3389/fnagi.2014.00140.

51. Spencer R.M.C., Ivry R.B., Zelaznik H. (2005) Role of the cerebellum in movements: control of timing or movement transition? Exp. Brain Res., 161: 383-396. DOI: 10.1007/s00221-004-2088-6.

52. Spirduso W., Francis K.L., MacRae P.G. (2005) Physical dimensions of aging. Human Kinetics Publ. Champaign.

53. Styns F., van Noorden L., Moelants D., Leman M. (2007) Walking on music. Hum. Mov. Sci., 26: 769-785. DOI: 10.1016/j.humov.2007.07.007.

54. Summers J.J. (1975) The role of timing in motor program representation. J. Motor Behav., 7: 229-241. DOI: 10.1080/00222895.1975.10735040.

55. Summers J.J., Pressing, J. (1994) Coordinating the two hands in polyrhythmic tapping. In: Swinnen S.P., Heuer H., Massion J., Casaer P. (eds.), Interlimb coordination: Neural, dynamical, and cognitive constraints. Academic Press, New York, pp. 571-591. DOI: 10.1016/B978-0-12679270-6.50031-0.

56. Thaut M., Rathbun J., Miller R. (1997) Music versus metronome timekeeper in a rhythmic motor task. Int. J. 
Arts Med., 5: 4-12. DOI: 10.1016/B978-0-12-6792706.50031-0.

57. Tierney A., Kraus N. (2015) Evidence for multiple rhythmic skills. PLoS One 10:e0136645.

58. Trehub S.E., Hannon E.E. (2009) Conventional rhythms enhance infants' and adults' perception of musical patterns. Cortex, 45: 110-118. DOI: 10.1016/j.cortex.2008.05.012.

59. Trehub S.E, Weiss M.W., Cirelli L.K. (2019). Musicality across the Lifespan. In: Rentfront P.J., Levitin D.J. (eds.) Foundations in Music Psychology: Theory and Research. The MIT Press, Cambridge, Massachusetts, London, England, p. 265.

60. Van Donkelaar P., Franks I.M. (1991) The preparation and initiation of simple rhythmical patterns. Hum. Mov. Sci., 10: 629-651. DOI: 10.1016/0167-9457[91]90020-X.

61. Vanneste S., Pouthas V., Wearden J.H. (2001) Temporal control of rhythmic performance: A comparison between young and old adults. Exp. Aging Res., 27: 83-102. DOI: 10.1080/03610730125798.

62. Wishart L.R., Lee T.D., Murdoch J.E., Hodges N.J. (2000) Effects of aging on automatic and effortful proc- esses in bimanual coordination. J. Gerontol. B-Psychol., 55: 85-94. DOI: 10.1093/geronb/55.2.P85.

63. Wood R.E. (1986) Task Complexity: Definition of the Construct. Organ. Behav. Hum. Dec., 37: 60-82. DOI: 10.1016/0749-5978(86)90044-0.

64. Zachopoulou E., Mantis K., (2001) The role of rhythmic ability on the forehand performance in tennis. Phys. Educ. Sport Pedagogy, 6: 117-126. DOI: 10.1080/1740898010060204.

65. Zelaznik H.N., Spencer R.M.C., Ivry R.B. (2000) Dissociation of explicit and implicit timing in repetitive tapping and drawing movements. J. Exp. Psychol. Human., 28: 575-588. DOI: 10.1037/0096-1523.28.3.575.

\section{Received 17.05.2021 \\ Accepted 10.11.2021}

(C) University of Physical Education, Warsaw, Poland 